\title{
Proceeding
}

6th INSHS International Christmas Sport Scientific Conference, 11-14 December 2011. International Network of Sport and

Health Science. Szombathely, Hungary

\section{The neurofeedback successfulness of sportsmen}

\author{
LARISA CHERAPKINA
}

Department of Anatomy, Physiology, Sport Medicine and Hygiene, Siberian State University of Physical Education and Sport, Russia

\begin{abstract}
Cherapkina L. The neurofeedback successfulness of sportsmen. J. Hum. Sport Exerc. Vol. 7, No. Proc1, pp. S116-S127, 2012. The research is devoted to studying of interconnection between successfulness of train to skill of regulation cerebrum bioelectric activity in alpha-band and to revealing of factors (sex, sport skill, general kinematic characteristic of executed motions) providing the distinctive changes of EEG. In first part of the work it's showed that cerebrum bioelectric activity gender differences is decreasing and reaction on eyes closing is getting more stereotyped if skill of sportsmen $(n=321)$ is growing. The sportsmen who practice acyclic substandard-variable types of sport have the least number of gender differences (by indexes of absolute and relative power in theta-, alpha- and beta-bands), and the greatest - sportsmen who practice cyclic types of sport. Less amounted reaction on the eyes closing is typical feature of sportswomen's cerebrum bioelectric activity regardless of sport skill and characteristic of executed motions. In second part of the work it is revealed that neurofeedback successfulness of sportsmen $(n=217)$ is being defined by totality of three factors $(F=2.805 ; P=0.027)$. Key words: SEX, SPORT SKILL, GENERAL KINEMATIC CHARACTERISTIC OF EXECUTED MOTIONS.
\end{abstract}

Corresponding author. Department of Anatomy, Physiology, Sport Medicine and Hygiene, Siberian State University of Physical Education and Sport, 644041,fl. 31, b. 11, s. Kharkovskay, c. Omsk, Russia

E-mail: kochelab@mail.ru

Phone: +79136143293

6th INSHS International Christmas Sport Scientific Conference, 11-14 December 2011. International Network of Sport and Health Science. Szombathely, Hungary

JOURNAL OF HUMAN SPORT \& EXERCISE ISSN 1988-5202

(c) Faculty of Education. University of Alicante

doi:10.4100/jhse.2012.7.Proc1.13 


\section{INTRODUCTION}

Growth of sport achievement orders necessity of searching of new methodologies for sport capacity for work and psychoemotional stability increasing. Neurofeedback is the most perspective in this direction. Numerous of the last years researches showed the neurofeedback positive influencing on functional condition of sportsmen and their performance efficiency (Baiova, 2003; Strizhkova et al., 2009; Visochin et al., 2005; Lopariov, 2009). At once majority of authors mention that neurophysiological mechanisms of the neurofeedback process are not studied enough (Konariova, 2005; Fedotchev, 2010; Mohovikova et al., 2005). At present successfulness and efficiency of the neurofeedback are differed. In Svyatogor's et al. (2000) opinion successfulness - it is changes of regulated parameter in specified direction (estimation of successfulness - it is way of defining of regulated parameter amount changes in specified direction), efficiency - it is positive changes of human organism functional condition under influencing of choose function directed regulation. Soroko and Musuraliev (1995) think, that neurofeedback successfulness is determined by initial EEG type, condition of examined person and by the individual plasticity of neurodynamical processes.

At works devoted to neurofeedback applying during sportsmen's training is showed that often efficiency of this methodology is determined by it successfulness (Cherapkina et al., 2006; Pogodaeva, 2001; Tristan et al., 2002). At the same time any authors note dependence of successfulness from gender differences (Tristan, 2005; Cherapkina, 2009), and other do not find this dependence (Kal'sina, 2002; Styopochkina et al., 2010). As it is known that male and female cerebrum structures have different ways since the earliest age. But a lot of authors mentioned that sportswomen have less amounted sex dimorphism indication in compare with sportsmen (Soboleva, 1999; Iordanskaya, 1999; Balakhnichev, 2007). It could influence on cerebrum functional activity and, as a consequence, on self-regulation of visceral functions by biofeedback using. Thereby unconsidered features of EEG pattern could be the reason of receiving of discrepant data about neurofeedback successfulness of different sex sportsmen. These features could be from one side the result of genetic predisposition and from other side the result of many years adaptation to physical loads.

The purpose of this work is the revealing of typical changes of sportsmen's EEG with different sex, sport skill, general kinematic characteristic of executed motions and the studying of it interconnection with successfulness of train to skill of regulation cerebrum bioelectric activity in alpha-band.

\section{MATERIAL AND METHODS}

In the research 30 sport specializations persons $(n=321)$ took part. According to common kinematical characteristic of executed motion 107 persons practiced acyclic substandard-variable types of sport, 123 persons - acyclic standard-variable types of sport and 91 persons - cyclic types of sport. Average age of examined persons was $19 \pm 0,1$ years old. Length of sport practice was 5 years and more. Dependently on sport skill all sportsmen were divided on three groups. First group consisted of low-skill sportsmen who had first and second adult grades ( $n=113)$, second - candidates to masters of sport ( $n=104)$, third - sportsmen with the highest skill (masters of sport, master of sports of international class and honoured masters of sport $(n=104))$. Among examined persons there are 125 female and 196 male. All sportsmen signed about participation agreement. 
The researches were holding with using software-hardware package "Boslab-alpha" (SRIMBB SB RAMS c. Novosibirsk, Russia). It consisted of multichannel interface BI-012 for computer monitoring, recording and reconstruction of EEG, kid of electrodes and software "BOSLAB". The bipolar disposal was used for the recording of cerebrum biopotentials. Electrodes were located according to international system "10-20" at frontal and parietal fields (F1, F2, P3, P4). Reference electrode was fasted to ear lobe and was connected to special plug of device. Electroconductive paste TEN-20 was used for laying of electrodes and it promoted the save fixing of electrodes during $30 \mathrm{~min}$. Skin of examined persons was hardly degreased by $70^{\circ}$ alcohol to improve the contact electrodes with scalp. For estimation of each sportsmen's neurofeedback successfulness percent of all training course successful sessions (by significant increasing of spectral EEG power in alpha-band comparing with first session indexes) was counted (Svyatogor, 2010).

The research consisted of two stages. At first the "background" cerebrum bioelectric activity of all examined persons ( $n=321$ ) was registered in trials with opened and closed eyes with following analysis of absolute and relative power in frequency bands theta $(4-8 \mathrm{~Hz})$, alpha $(8-13 \mathrm{~Hz})$, beta $(13-20 \mathrm{~Hz})$ activity. The duration of each trial was 5 min.

At second stage 217 sportsmen (123 male and 94 female) had 15 days neurofeedback course directed to increasing EEG power in alpha-band by Pogadaeva's methodology (2001). The sessions were holding 1 time per day. Pre-briefing and subsidiary explanation about relaxation during session as obligatory term for successful biofeedback was held before training (Schwartz, 1998). The sportsmen used different methods to prevent appearance of anxiety feel during training (Bugaiova et al., 1993; Crider, 1998; Thomas et al., 1991). It was suggested psycho-muscular train (feel of warm in the extremities), creation of positive images (landscapes with green and blue colors) and also free methods. During neurofeedback session which continues $25-30$ min, sportsmen were located in chair with closed eyes. Their task was to achieve more frequent sound signal of feedback which appeared if alpha-rhythm power was higher than threshold mean during $0.2 \mathrm{~s}$. The threshold of appearance of feedback signal was being fixed before beginning session thus to alpha-rhythm power could be higher than initial in 30\% cases. In the case if during session the alpha-rhythm power was decreasing stably the threshold of appearance of feedback signal was decreased also (by hands).

Statistical analysis of received data was carried out with using of software product SPSS 13.0. Parametrical and nonparametrical (dependently of distribution character) methods was being used for description. The normalcy of distribution was determined by skewness and kurtosis criterions. Maximum probability of errors (minimum level of significance) in the interpretation of statistical data was mean $p<0.05$.

\section{RESULTS}

Comparative analysis of studied EEG indexes of different skill sportsmen showed that among low-skill sportsmen there are significant gender differences (Tables 1 and 2). At the sportswomen's left hemisphere quantity of absolute spectral power in theta-band in tail with opened eyes was the largest in compare with sportsmen and in alpha-band in trail with closed eyes was the least (Table 1). Sportswomen had less than sportsmen spectral power in alpha-band at right hemisphere in a state of opened and closed eyes and in beta-band in a state of closed eyes. In all studied bands as sportswomen so sportsmen had significant left hemisphere asymmetry. Reaction to eyes closing was observed only of sportsmen and it was demonstrated by increasing of power in all studied bands at right hemisphere and in alpha-band at left hemisphere. Differences between relative power amount of low-skill sportsmen and low-skill sportswomen were not revealed only in alpha-band of right hemisphere in a state of closed eyes (Table 2). Other 
sportsmen's indexes as opposed to sportswomen were described by less relative power in theta-band and by more in alpha- and beta-bands. By amount of relative spectral power the interhemispheric asymmetries were revealed only of sportswomen. It was demonstrated by more marked power in theta-band of left hemisphere in a state of opened eyes and in alpha-band of right hemisphere in a state of closed eyes. The increasing of power in alpha-band of right hemisphere was common for both sportswomen and sportsmen. In addition the decreasing of relative power in theta-band of both hemispheres, the increasing in alphaband and decreasing in beta-band of left hemisphere of sportsmen was mentioned.

The high-skill sportsmen (CMS, MS, MSIC, HMS) did not have gender differences by amounts of absolute and relative spectral power (Tables 1 and 2). All examined persons had left hemisphere predominance of spectral power in theta-, alpha- and beta-bands. Interhemispheric differences by amount of relative power were indicated only of candidates to masters of sport. The sportsmen had predominated power of left hemisphere in theta-band in a state of opened and closed eyes and of right hemisphere in alpha-band with closed eyes. The sportswomen had predominated left hemisphere over right in theta-band with closed eyes. In group of candidate to masters of sport the eyes closing provoked the increasing of power in alphaband of both sportswomen's and sportsmen's left hemisphere. But in addition sportsmen had power increasing in theta-band of left hemisphere, alpha-band of right hemisphere and in beta-band of both hemispheres. The both sex sportsmen had the decreasing of relative power amount in theta-band of right hemisphere and increasing in alpha-band of both hemispheres as reaction on eyes closing. Sportsmen had decreasing of relative power amount at left hemisphere during eyes closing.

In the group of different sex highest skill sportsmen there is more similar response on eyes closing. The sportswomen and sportsmen entered at this group had growth of spectral power in alpha-band during eyes closing (Table 1). In addition sportsmen had increasing of power in beta-band of left hemisphere. On eyes closing as sportswomen so sportsmen had similar changes of relative power in both hemispheres which were demonstrated by decreasing of power in theta-band and by growth in alpha-band (Table 2). 
Table 1. The gender differenees between sportsmen with different skill by quantity of spectral power in theta-, alpha- and beta-band at the studied field of cerebral cortex

\begin{tabular}{|c|c|c|c|c|c|c|}
\hline \multirow{2}{*}{$\begin{array}{l}\text { Frequen- } \\
\text { cy band }\end{array}$} & \multirow{2}{*}{$\begin{array}{l}\text { Hemis- } \\
\text { phere }\end{array}$} & \multirow{2}{*}{ Groups } & \multicolumn{2}{|c|}{ Opened eyes } & \multicolumn{2}{|c|}{ Closed eyes } \\
\hline & & & $\mathrm{M} \pm \mathrm{m}$ & $\mathrm{P}$ & $\mathrm{M} \pm \mathrm{m}$ & $\mathrm{P}$ \\
\hline \multicolumn{7}{|c|}{ I group (I, II adult grades) } \\
\hline \multirow{4}{*}{ theta } & \multirow{2}{*}{ left } & female & $6,416 \pm 0,260^{\wedge}$ & \multirow{2}{*}{0,025} & $6,332 \pm 0,250^{\wedge}$ & \multirow{2}{*}{0,059} \\
\hline & & male & $6,042 \pm 0,242^{\wedge}$ & & $6,192 \pm 0,297^{\wedge}$ & \\
\hline & \multirow{2}{*}{ right } & female & $4,168 \pm 0,153$ & \multirow{2}{*}{0,988} & $4,217 \pm 0,196$ & \multirow{2}{*}{0,335} \\
\hline & & male & $4,194 \pm 0,129$ & & $4,474 \pm 0,182^{*}$ & \\
\hline \multirow{4}{*}{ alpha } & \multirow{2}{*}{ left } & female & $2,981 \pm 0,099^{\wedge}$ & \multirow{2}{*}{0,127} & $3,150 \pm 0,166^{\wedge}$ & \multirow{2}{*}{0,041} \\
\hline & & male & $3,320 \pm 0,106^{\wedge}$ & & $3,710 \pm 0,153^{\wedge}$ & \\
\hline & \multirow{2}{*}{ right } & female & $2,178 \pm 0,144$ & \multirow{2}{*}{0,054} & $2,503 \pm 0,262$ & \multirow{2}{*}{0,015} \\
\hline & & male & $2,610 \pm 0,138$ & & $3,033 \pm 0,181^{*}$ & \\
\hline \multirow{4}{*}{ beta } & \multirow{2}{*}{ left } & female & $3,329 \pm 0,100^{\wedge}$ & \multirow{2}{*}{0,082} & $3,395 \pm 0,163^{\wedge}$ & \multirow{2}{*}{0,065} \\
\hline & & male & $3,812 \pm 0,135^{\wedge}$ & & $3,962 \pm 0,187^{\wedge}$ & \\
\hline & \multirow{2}{*}{ right } & female & $2,472 \pm, 152$ & 0.076 & $2,476 \pm 0,171$ & 0010 \\
\hline & & male & $3,427 \pm 0,465$ & $0,0 \%$ & $3,626 \pm 0,468^{*}$ & 0,019 \\
\hline & & & 2 group (CMS & & & \\
\hline & leff & female & $6,145 \pm 0,300^{\wedge}$ & 0848 & $6,389 \pm 0,301^{\wedge}$ & 0675 \\
\hline theta & Ient & male & $6,016 \pm 0,215^{\wedge}$ & & $6,356 \pm 0,298^{\wedge *}$ & 0,073 \\
\hline theta & right & female & $4,305 \pm 0,191$ & 0765 & $4,225 \pm 0,156$ & 0,643 \\
\hline & ngnt & male & $4,389 \pm 0,245$ & $0, / 05$ & $4,389 \pm 0,205$ & 0,043 \\
\hline & left & female & $3,047 \pm 0,092^{\wedge}$ & 0.408 & $3,389 \pm 0,124^{\wedge *}$ & 0604 \\
\hline alnha & ICIt & male & $3,306 \pm 0,141^{\wedge}$ & 0,498 & $3,946 \pm 0,281^{\wedge *}$ & 0,094 \\
\hline alpna & rioht & female & $2,373 \pm 0,137$ & 0.692 & $2,556 \pm 0,168$ & 0268 \\
\hline & ngnt & male & $2,648 \pm 0,188$ & 0,092 & $3,257 \pm 0,373^{*}$ & 0,208 \\
\hline & left & female & $3,261 \pm 0,094^{\wedge}$ & 0071 & $3,436 \pm 0,126^{\wedge}$ & 0258 \\
\hline beta & & male & $3,729 \pm 0,173^{\wedge}$ & & $4,172 \pm 0,375^{\wedge *}$ & \\
\hline Deta & riaht & female & $2,511 \pm 0,116$ & $0>04$ & $2,567 \pm 0,129$ & 0223 \\
\hline & ngmi & male & $2,934 \pm 0,151$ & 0,204 & $3,092 \pm 0,187^{*}$ & 0,223 \\
\hline & & & roup (MS, MSIC & MS) & & \\
\hline & left & female & $5,497 \pm 0,220^{\wedge}$ & 0.733 & $5,487 \pm 0,202^{\wedge}$ & 0784 \\
\hline theta & leit & male & $5,625 \pm 0,221^{\wedge}$ & & $5,674 \pm 0,210^{\infty}$ & \\
\hline & rioht & female & $4,134 \pm 0,144$ & 0711 & $4,264 \pm 0,164$ & 0268 \\
\hline & & male & $4,233 \pm 0,164$ & 0,111 & $4,125 \pm 0,154$ & 0,208 \\
\hline & left & female & $3,430 \pm 0,220^{\wedge}$ & 0.183 & $3,704 \pm 0,245^{\wedge *}$ & 0305 \\
\hline alpha & ICII & male & $3,304 \pm 0,222^{\wedge}$ & 0,103 & $3,707 \pm 0,285^{\wedge \bullet}$ & 0,093 \\
\hline & rioht & female & $2,780 \pm 0,198$ & 0.206 & $3,123 \pm 0,224^{*}$ & 0.186 \\
\hline & ngnt & male & $2,590 \pm 0,187$ & 0,206 & $2,899 \pm 0,237^{*}$ & 0,180 \\
\hline & left & female & $3,752 \pm 0,177^{\wedge}$ & 0.280 & $3,904 \pm 0,192^{\wedge}$ & 0.556 \\
\hline heta & & male & $3,700 \pm 0,187^{\wedge}$ & 0,200 & $3,858 \pm 0,210^{\wedge *}$ & \\
\hline & right & fermale & $3,003 \pm 0,184$ & 0.386 & $3,265 \pm 0,244$ & 0.276 \\
\hline & & male & $2,762 \pm 0,142$ & & $2,809 \pm 0,162$ & \\
\hline
\end{tabular}

$\wedge$ - differences in comparison with right hemisphere with $\mathrm{P}<0,05 ;{ }^{*}$ - differences in comparison with opened eyes condition with $\mathrm{P}<0,05$. 
Table 2. The gender differences between sportsmen with different skill by the relative quantity of spectral power in theta-, alpha- and beta-band at the study field of cerebrum cortex

\begin{tabular}{|c|c|c|c|c|c|c|}
\hline \multirow{2}{*}{$\begin{array}{l}\text { Frequen- } \\
\text { cy band }\end{array}$} & \multirow{2}{*}{$\begin{array}{l}\text { Hemis- } \\
\text { phere }\end{array}$} & \multirow{2}{*}{ Groups } & \multicolumn{2}{|c|}{ Opened eyes } & \multicolumn{2}{|c|}{ Closed eyes } \\
\hline & & & $\mathrm{M} \pm \mathrm{m}$ & $\mathrm{P}$ & $\mathrm{M} \pm \mathrm{m}$ & $\mathrm{P}$ \\
\hline \multicolumn{7}{|c|}{1 group (I, II adult grades) } \\
\hline \multirow{4}{*}{ theta } & \multirow{2}{*}{ left } & female & $49,975 \pm 1,060^{\wedge}$ & \multirow{2}{*}{0,003} & $49,168 \pm 1,242$ & \multirow{2}{*}{0,002} \\
\hline & & male & $45,584 \pm 0,846$ & & $44,600 \pm 0,887^{*}$ & \\
\hline & \multirow{2}{*}{ right } & female & $48,070 \pm 0,859$ & \multirow{2}{*}{0,003} & $47,352 \pm 1,025$ & \multirow{2}{*}{0,010} \\
\hline & & male & $44,124 \pm 0,992$ & & $43,204 \pm 1,011^{*}$ & \\
\hline \multirow{4}{*}{ alpha } & \multirow{2}{*}{ left } & female & $23,428 \pm 0,393$ & \multirow{2}{*}{0,004} & $24,253 \pm 0,657^{\wedge}$ & \multirow{2}{*}{0,001} \\
\hline & & male & $25,267 \pm 0,393$ & & $26,808 \pm 0,512^{*}$ & \\
\hline & \multirow{2}{*}{ right } & female & $24,231 \pm 0,510$ & \multirow{2}{*}{0,041} & $25,819 \pm 0,893^{*}$ & \multirow{2}{*}{0,136} \\
\hline & & male & $25,627 \pm 0,521$ & & $27,078 \pm 0,640^{\circ}$ & \\
\hline \multirow{4}{*}{ beta } & \multirow{2}{*}{ left } & female & $26,597 \pm 0,889$ & \multirow{2}{*}{0,009} & $26,579 \pm 0,988$ & \multirow{2}{*}{0,011} \\
\hline & & male & $29,150 \pm 0,658$ & & $28,592 \pm 0,568^{*}$ & \\
\hline & \multirow{2}{*}{ right } & female & $27,700 \pm 0,707$ & & $26,829 \pm 0.494$ & \\
\hline & & male & $30,249 \pm 0,999$ & 0,024 & $29,718 \pm 0,908$ & 0,031 \\
\hline & & & 2 group (CMS & & & \\
\hline & left & female & $48,697 \pm 1,155$ & 0128 & $47,936 \pm 1,178^{\wedge}$ & 0123 \\
\hline theta & lent & male & $46,309 \pm 0,958^{\wedge}$ & 0,120 & $45,299 \pm 1,065^{\wedge}$ & 0,125 \\
\hline Inetas & riobt & female & $47,130 \pm 1,085$ & 0266 & $45,933 \pm 1,002^{*}$ & 0162 \\
\hline & rignt & male & $44,404 \pm 1,086$ & 0,200 & $42,663 \pm 1,130^{*}$ & 0,102 \\
\hline & left & female & $24,624 \pm 0,492$ & 0848 & $25,742 \pm 0,572^{*}$ & 0954 \\
\hline alnh & ient & male & $25,186 \pm 0,559$ & 0,848 & $26,770^{\wedge} \pm 0.818^{*}$ & 0,934 \\
\hline alpha & & female & $25,427 \pm 0,747$ & 0.954 & $26,678 \pm 0,861^{*}$ & 0,312 \\
\hline & right & male & $25,611 \pm 0,604$ & 0,954 & $28,100 \pm 0,897^{*}$ & 0,312 \\
\hline & left & female & $26,678 \pm 0,819$ & 0.057 & $26,322 \pm 0,800$ & 0.094 \\
\hline heta & lent & male & $28,504 \pm 0,630$ & $0,03 \pi$ & $27,932 \pm 0,655$ & 0,094 \\
\hline & right & female & $27,443 \pm 0,728$ & 0.093 & $27,389 \pm 0,656$ & 0.552 \\
\hline & rignt & male & $29,986 \pm 0,823$ & 0,090 & $29,237 \pm 0,864$ & 0,352 \\
\hline & & & roup (MS, MSIC & MS) & & \\
\hline & left & female & $43,831 \pm 1,323$ & 0.616 & $42,775 \pm 1,337^{*}$ & 0518 \\
\hline theta & Itit & male & $45,032 \pm 1,150$ & 0,010 & $43,987 \pm 1,251^{*}$ & סוקוס, \\
\hline tneta & right & female & $42,350 \pm 1,550$ & 0286 & $41,402 \pm 1,618^{*}$ & 0.500 \\
\hline & ngm & male & $45,310 \pm 1,047$ & 0,260 & $43,654 \pm 1,137^{*}$ & 0,590 \\
\hline & left & female & $26,452 \pm 0,786$ & 0653 & $27,424 \pm 0,834^{*}$ & 0535 \\
\hline alnha & lent & male & $25,692 \pm 0,642$ & 0,033 & $27,106 \pm 0,909^{*}$ & 0,559 \\
\hline & & female & $27,405 \pm 0,783$ & 0.151 & $28,713 \pm 0,990^{*}$ & 0.375 \\
\hline & nghl & male & $26,000 \pm 0,662$ & 0,131 & $27,955 \pm 0,926^{*}$ & $0,3 / 5$ \\
\hline & left & female & $29,716 \pm 0,924$ & 0.790 & $29.800 \pm 0,929$ & 0.644 \\
\hline beta & ICIt & male & $29,276 \pm 0,724$ & 0,790 & $28,908 \pm 0,737$ & $0,0+4$ \\
\hline & right & female & $30,245 \pm 0,992$ & 0.724 & $29,885 \pm 1,016$ & 0.960 \\
\hline & ngmit & male & $28,690 \pm 0,614$ & 0,724 & $28,392 \pm 0,618$ & 0,900 \\
\hline
\end{tabular}

$\wedge$ - differences in comparison with right hemisphere with $\mathrm{P}<0,05 ; \cdot$ - differences in comparison with opened eyes condition with $\mathrm{P}<0,05$. 
The studying of gender differences in sportsmen's group differed by kinematic characteristic of executed motions showed that regardless of sex left hemisphere was predominated over right by absolute power in all studied bands (Table 3). Differences between sportswomen and sportsmen practicing acyclic substandard-variable types of sport by amount of absolute spectral power were not revealed. The sportswomen's relative power was higher in theta-band of right hemisphere with opened and closed eyes and lower in alpha-band of both hemispheres with closed eyes (Tables 3 and 4). The eyes closing by sportsmen provoked growth of spectral power in theta- and beta-bands of right hemisphere and in alphaband of both hemispheres. The sportsmen had more significant changes of relative power than sportswomen during eyes closing. Particularly they had the decreasing of power in theta-band and the increasing in alpha-band of both hemispheres. Also growth of power was observed in beta-band of left hemisphere. The eyes closing by sportswomen provoked only the decreasing of power in theta-band at left hemisphere.

In group of sportsmen practicing acyclic standard-variable types of sport differences between sportswomen and sportsmen were revealed only in alpha-band in trail with opened eyes. The sportswomen had higher amount of absolute spectral power at left hemisphere and of relative power at right hemisphere (Tables 3 and 4). Sportswomen's and sportsmen's reaction on the eyes closing was described by growth of absolute power in alpha-band at both hemispheres. Also sportsmen had increasing of power in beta-band at both hemispheres. Relative power decreased in theta-band and increased in alpha-band during eyes closing of both sex persons. In addition sportswomen had decreasing of relative power in beta-band at right hemisphere.

In a state of opened and closed eyes in sportsmen's group practicing cyclic types of sport sportswomen were dissimilar with sportsmen by less amount of absolute power in alpha- and beta-bands and by higher relative power in theta-band at left hemisphere (Tables 3 and 4). Only sportsmen had changes of absolute power at a state of closed eyes which were described by growth in alpha-band at both hemispheres and in beta-band at left hemisphere. In a state of closed eyes relative power of both sex sportsmen decreased in theta-band at right hemisphere and increased in alpha-band at both hemispheres. Also sportsmen had decreasing of relative power in theta-band at left hemisphere and sportswomen - in beta-band at left hemisphere. 
Table 3. The gender differences between sportsmen are dissimilar by kinematical characteristics of executed motions by quantity of spectral power in theta-, alpha- and beta-band at the study field of cerebrum cortex

\begin{tabular}{|c|c|c|c|c|c|c|}
\hline \multirow{2}{*}{$\begin{array}{l}\text { Frequen- } \\
\text { cy band }\end{array}$} & \multirow{2}{*}{$\begin{array}{c}\text { Hemis- } \\
\text { phere }\end{array}$} & \multirow{2}{*}{ Groups } & \multicolumn{2}{|c|}{ Opened eyes } & \multicolumn{2}{|c|}{ Closed eyes } \\
\hline & & & $\mathrm{M} \pm \mathrm{m}$ & $\mathrm{P}$ & $\mathrm{M} \pm \mathrm{m}$ & $\mathrm{P}$ \\
\hline \multicolumn{7}{|c|}{ Acyclic substandard-variable types of sport } \\
\hline \multirow{4}{*}{ theta } & \multirow{2}{*}{ left } & female & $5,954 \pm 0,336^{\wedge}$ & \multirow{2}{*}{0,565} & $6,084 \pm 0,376^{\wedge}$ & \multirow{2}{*}{0,707} \\
\hline & & male & $5,997 \pm 0,217^{\wedge}$ & & $6,147 \pm 0,267^{\wedge}$ & \\
\hline & \multirow{2}{*}{ right } & female & $4,445 \pm 0,238$ & \multirow{2}{*}{0,362} & $4,492 \pm 0,318$ & \multirow{2}{*}{0,914} \\
\hline & & male & $4,151 \pm 0,130$ & & $4,408 \pm 0,184^{*}$ & \\
\hline \multirow{4}{*}{ alpha } & \multirow{2}{*}{ left } & female & $2,950 \pm 0,150^{\wedge}$ & \multirow{2}{*}{0,094} & $3,209 \pm 0,201^{\wedge}$ & \multirow{2}{*}{0,136} \\
\hline & & male & $3,358 \pm 0,108^{\wedge}$ & & $3,896 \pm 0,193^{\wedge *}$ & \\
\hline & \multirow{2}{*}{ right } & female & $2,369 \pm 0,216$ & \multirow{2}{*}{0.523} & $2,614 \pm 0,329$ & \multirow{2}{*}{0,121} \\
\hline & & male & $2,699 \pm 0,159$ & & $3,398 \pm 0,304^{*}$ & \\
\hline \multirow{4}{*}{ beta } & \multirow{2}{*}{ left } & female & $3,348 \pm 0,147^{\wedge}$ & \multirow{2}{*}{0,214} & $3,691 \pm 0,276^{\wedge}$ & \multirow{2}{*}{0,637} \\
\hline & & male & $3,787 \pm 0,127^{\wedge}$ & & $3,921 \pm 0,168^{\wedge}$ & \\
\hline & \multirow{2}{*}{ right } & female & $2,677 \pm 0,214$ & 0.629 & $2,810 \pm 0,265$ & 0.422 \\
\hline & & male & $3,196 \pm 0,340$ & $0,0<9$ & $3,540 \pm 0,406^{*}$ & \\
\hline & & Acyc & andard-variable t & es of $s_{1}$ & & \\
\hline & left & female & $5,946 \pm 0,197^{\wedge}$ & 0.314 & $5,914 \pm 0,173^{\wedge}$ & 0.350 \\
\hline thets & lent & male & $5,704 \pm 0,252^{\wedge}$ & 0,514 & $5,983 \pm 0,359^{\wedge}$ & 0,559 \\
\hline totat & right & female & $4,173 \pm 0,120$ & 0.847 & $4,297 \pm 0,134$ & 0357 \\
\hline & rignt & male & $4,221 \pm 0,146$ & 0,847 & $4,187 \pm 0,159$ & $0,5,3$ \\
\hline & left & female & $3,355 \pm 0,134^{\wedge}$ & 0.014 & $3,665 \pm 0,174^{\wedge \star}$ & 0.081 \\
\hline alnha & lent & male & $3,077 \pm 0,132^{\wedge}$ & 0,014 & $3,570 \pm 0,271^{\wedge *}$ & \\
\hline & right & female & $2,638 \pm 0,129$ & 0.162 & $3,004 \pm 0,179^{*}$ & 0.180 \\
\hline & rignt & male & $2,384 \pm 0,120$ & 0,102 & $2,677 \pm 0,163^{*}$ & 0,180 \\
\hline & & female & $3,654 \pm 0,126^{\wedge}$ & 0.443 & $3,731 \pm 0,125^{\wedge}$ & 0.451 \\
\hline bota & left & male & $3,648 \pm 0,191^{\wedge}$ & 0,443 & $4,116 \pm 0,443^{\wedge *}$ & 0,451 \\
\hline Deta & rioht & female & $2,821 \pm 0,134$ & & $2,930 \pm 0,153$ & 0.703 \\
\hline & right & male & $2,749 \pm 0,132$ & 0,801 & $2,842 \pm 0,165^{*}$ & \\
\hline & & & Cyclic types of s & & & \\
\hline & left & female & $6,003 \pm 0,324^{\wedge}$ & 0.932 & $6,158 \pm 0,334^{\wedge}$ & 0.948 \\
\hline theta & leit & male & $6,004 \pm 0.219^{\wedge}$ & 0,932 & $6,161 \pm 0,209^{\wedge}$ & \\
\hline & right & female & $4,108 \pm 0,187$ & 0.387 & $3,987 \pm 0,138$ & 0.137 \\
\hline & ngnt & male & $4,510 \pm 0,284$ & 0,381 & $4,422 \pm 0,195$ & 0,137 \\
\hline & left & female & $2,976 \pm 0,201^{\wedge}$ & & $3,159 \pm 0,191^{\wedge}$ & 0.044 \\
\hline glnha & leit & male & $3,465 \pm 0,223^{\wedge}$ & 0,032 & $3,832 \pm 0,264^{\wedge *}$ & \\
\hline & right & female & $2,233 \pm 0,212$ & 0.075 & $2,395 \pm 0,220$ & 0,099 \\
\hline & right & male & $2,719 \pm 0,205$ & $0,0 / 5$ & $2,938 \pm 0,221^{*}$ & 0,099 \\
\hline & $\mathrm{lcft}$ & female & $3,209 \pm 0,129^{\wedge}$ & 0.033 & $3,338 \pm 0,195^{\wedge}$ & 0,006 \\
\hline beta & & male & $3,804 \pm 0,190^{\wedge}$ & & $4,022 \pm 0,221^{\wedge}$ & \\
\hline & right & female & $2,465 \pm 0,166$ & 0.085 & $2,605 \pm 0,255$ & 0,073 \\
\hline & rignt & male & $3,230 \pm 0,421$ & 0,003 & $3,104 \pm 0,262$ & \\
\hline
\end{tabular}

$\wedge$ - differences in comparison with right hemisphere with $\mathrm{P}<0,05 ;{ }^{*}$ - differences in comparison with opened eyes condition with $\mathrm{P}<0,05$. 
Table 4. The gender differences between sportsmen are dissimilar by kinematical characteristics of executed motions by the relative quantity of spectral power in theta-, alpha- and beta-band at the study field of cerebrum cortex

\begin{tabular}{|c|c|c|c|c|c|c|}
\hline \multirow{2}{*}{$\begin{array}{l}\text { Frequen- } \\
\text { cy band }\end{array}$} & \multirow{2}{*}{$\begin{array}{c}\text { Hemis- } \\
\text { phere }\end{array}$} & \multirow{2}{*}{ Groups } & \multicolumn{2}{|c|}{ Opened eyes } & \multicolumn{2}{|c|}{ Closed eyes } \\
\hline & & & $\mathrm{M} \pm \mathrm{m}$ & $\mathrm{P}$ & $\mathrm{M} \pm \mathrm{m}$ & $\mathbf{P}$ \\
\hline \multicolumn{7}{|c|}{ Acyclic substandard-variable types of sport } \\
\hline \multirow{4}{*}{ theta } & \multirow{2}{*}{ left } & female & $48,310 \pm 1,46$ & \multirow{2}{*}{0,174} & $46,811 \pm 1,656^{*}$ & \multirow{2}{*}{0,276} \\
\hline & & male & $45,538 \pm 0,849$ & & $44,355 \pm 0,918^{\wedge *}$ & \\
\hline & \multirow{2}{*}{ right } & female & $47,596 \pm 1,276$ & \multirow{2}{*}{0,029} & $46,578 \pm 1,553$ & \multirow{2}{*}{0,053} \\
\hline & & male & $43,592 \pm 0,952$ & & $41,871 \pm 1,010^{*}$ & \\
\hline \multirow{4}{*}{ alpha } & \multirow{2}{*}{ left } & female & $24,058 \pm 0,698$ & \multirow{2}{*}{0,087} & $24,653 \pm 0,844$ & \multirow{2}{*}{0,026} \\
\hline & & male & $25,550 \pm 0,458$ & & $27,554 \pm 0,678^{*}$ & \\
\hline & \multirow{2}{*}{ right } & female & $24,392 \pm 0,748$ & \multirow{2}{*}{0,110} & $25,386 \pm 1,252$ & \multirow{2}{*}{0,020} \\
\hline & & male & $26,146 \pm 0,548$ & & $28,467 \pm 0,778^{*}$ & \\
\hline \multirow{4}{*}{ beta } & \multirow{2}{*}{ left } & female & $27,632 \pm 1,176$ & \multirow{2}{*}{0,260} & $28,536 \pm 1,470$ & \multirow{2}{*}{0,859} \\
\hline & & male & $28,912 \pm 0,579$ & & $28,091 \pm 0,515^{*}$ & \\
\hline & \multirow{2}{*}{ right } & female & $28,012 \pm 1,109$ & & $28,036 \pm 0,686$ & 0.911 \\
\hline & & male & $30,262 \pm 0,814$ & 0,063 & $29,663 \pm 0,876$ & 0,911 \\
\hline & & Acy & tandard-variable & es of st & & \\
\hline & left & female & $45,886 \pm 1,037$ & 0714 & $44,862 \pm 1,021^{*}$ & 0.768 \\
\hline thet & lent & male & $45,806 \pm 0,920$ & $0,7 / 4$ & $44,752 \pm 0,992^{*}$ & $0, / 68$ \\
\hline tneta & rioht & female & $43,769 \pm 1,154$ & 0308 & $42,995 \pm 1,181^{*}$ & 0.761 \\
\hline & rignt & male & $45,806 \pm 0,917$ & 0,298 & $44,326 \pm 1,022^{*}$ & 0,701 \\
\hline & left & female & $25,730 \pm 0,530^{\wedge}$ & 0,400 & $27,002 \pm 0,620^{\wedge}$ & 0.338 \\
\hline & lent & male & $24,750 \pm 0,363$ & 0,409 & $26,083 \pm 0,563^{\wedge *}$ & 0,358 \\
\hline alpha & rioht & female & $26,966 \pm 0,596$ & 0.009 & $28,586 \pm 0,742^{*}$ & 0096 \\
\hline & rignt & male & $25,098 \pm 0,475$ & 0,029 & $26,951 \pm 0,741^{*}$ & 0,096 \\
\hline & & female & $28,385 \pm 0,754$ & & $28,137 \pm 0,708$ & 0.139 \\
\hline hoto & left & malc & $29,445 \pm 0,704$ & $0,1 / 5$ & $29,165 \pm 0,703$ & 0,139 \\
\hline Deta & & female & $29,265 \pm 0,759$ & & $28,419 \pm 0,731^{*}$ & 0.180 \\
\hline & right & male & $29.096 \pm 0,624$ & 0,521 & $28,723 \pm 0,640$ & 0,189 \\
\hline & & & Cyclic types of & & & \\
\hline & left & female & $48,821 \pm 1,414$ & 0043 & $48,518 \pm 1,579^{\wedge}$ & 0.045 \\
\hline theta & left & male & $45,753 \pm 1,164$ & 0,043 & $45,069 \pm 1,259^{*}$ & $0,0+3$ \\
\hline theta & right & female & $47,692 \pm 1,241$ & 0166 & $46,377 \pm 1,312^{*}$ & 0143 \\
\hline & right & male & $44,800 \pm 1,236$ & 0,106 & $44,020 \pm 1,183^{*}$ & $0,1+3$ \\
\hline & left & female & $24,163 \pm 0,738$ & 0.067 & $24,780 \pm 0,754^{*}$ & 0.189 \\
\hline aln & left & male & $25,649 \pm 0,691$ & $0,06 /$ & $26,594 \pm 0,870^{*}$ & 0,189 \\
\hline alpha & & female & $24,579 \pm 0,792$ & 0172 & $25,703 \pm 0,981^{*}$ & 0223 \\
\hline & right & male & $25,666 \pm 0,696$ & 0,172 & $27,059 \pm 0,824^{*}$ & 0,223 \\
\hline & left & female & $27,016 \pm 0,946$ & 0108 & $26,702 \pm 1,053^{*}$ & 0.077 \\
\hline beta & left & male & $28,598 \pm 0,771$ & 0,108 & $28,337 \pm 0,781$ & $0,07 / 7$ \\
\hline Deta & riobt & female & $27,729 \pm 0,781$ & 0.444 & $27,920 \pm 0,887$ & 0,307 \\
\hline & right & male & $29,534 \pm 1,130$ & 0,444 & $28,921 \pm 0,858$ & 0,307 \\
\hline
\end{tabular}

${ }^{\wedge}$ - differences in comparison with right hemisphere with $\mathrm{P}<0,05 ;{ }^{*}$ - differences in comparison with opened eyes condition with $\mathrm{P}<0,05$. 
Analysis of received data during examination of sportsmen $(n=217)$ having neurofeedback course showed that they did not have gender differences by indexes changes of spectral power during training. The average means for 2-15 sessions of power in alpha- and beta-bands were significantly higher than equal indexes of first session of both sex persons (Table 5). At the same time sportsmen had successfulness index equaled $41 \pm 2.9 \%$, and sportswomen $-43 \pm 2.7 \%$ ( $P>0.05)$.

But multivariate analysis of variance with preliminary test on homogeneity of variance using Levene's test $(F=1.440 ; P>0.05)$ showed that combination of three factors influence on neurofeedback successfulness: sex, sport specialization and skill ( $F=2.805 ; P=0.027)$ (Figure 1) As a covariant which promoted $21 \%$ of dispersion of training successfulness $(2=0.212)$ was expressed in presents number of sessions during which age coefficient was higher than initial amount for $15 \%$. Among low-skill persons the sportsmen had similar neurofeedback successfulness regardless of character of executed motions. Sportswomen otherwise had significant differences by lower neurofeedback successfulness of sportswomen practicing cyclic types of sport. In groups of high-skill persons (CMS, MS, MSIC, HMS) neurofeedback successfulness of sportswomen and sportsmen significantly differed dependently on kinematical characteristic of executed motions. Also in all tree skilled groups successfulness of persons practicing cyclic types of sport had more significant gender differences. Sportsmen practicing acyclic standardvariable types of sport in groups of low-skill persons and of candidate to masters of sport and also sportsmen practicing acyclic substandard-variable types of sport had less evident gender differences.

Table 5. The changes of spectral power of cerebrum bioelectrical activity of sportsmen with different sex during the neurofeedback course $(\mathrm{M} \pm \mathrm{m}), \mathrm{mcV}^{2}$

\begin{tabular}{|l|c|c|c|}
\hline \multirow{2}{*}{ Spectral power } & \multicolumn{3}{|c|}{ Frequently band } \\
\cline { 2 - 3 } & theta & alpha & beta \\
\hline & \multicolumn{2}{|c|}{ male $(\mathrm{n}=123)$} \\
\hline Mean of first session & $5,863 \pm 0,137$ & $\begin{array}{c}3,507 \pm 0,113 \\
\mathrm{P}<0,001\end{array}$ & $\begin{array}{c}3,729 \pm 0,117 \\
\mathrm{P}<0,001 \\
3,747 \pm 0,058\end{array}$ \\
\hline $\begin{array}{l}\text { Mean of 2-15 sessions } \\
\text { of training }\end{array}$ & $5,884 \pm 0,084$ & $\begin{array}{c}3,694 \pm 0,105 \\
\text { female ( } \mathrm{n}=94)\end{array}$ \\
\hline Mean of first session & $5,945 \pm 0,126$ & $\begin{array}{c}3,479 \pm 0,128 \\
\mathrm{P}<0,001\end{array}$ & $\begin{array}{c}3,638 \pm 0,103 \\
\mathrm{P}<0,002\end{array}$ \\
\hline $\begin{array}{l}\text { Mean of 2-15 sessions } \\
\text { of training }\end{array}$ & $5,862 \pm, 0931$ & $3,801 \pm 0,109$ & $3,802 \pm, 0635$ \\
\hline
\end{tabular}

\section{DISCUSSION}

As earlier was mentioned, the neurofeedback applying pass ahead it's theoretical basis (Svyatogor, 2000; Konariova, 2005; Fedotchev, 2010). The mechanisms used by human for regulation of EEG rhythms power still are not clear. The held research showed that evident gender differences are typical for low-skill sportsmen. With growth of sport skill gender differences get smoothed and reaction on eyes closing gets more similar. Dependently on kinematical characteristic of executed motions sportsmen practicing acyclic standard-variable types of sport have the least amount of gender differences (by indexes of absolute and relative power in alpha-band and reactive changes in beta-band). They are followed by sportsmen 
practicing acyclic substandard-variable types of sport who have gender differences by amount of relative power in alpha- and theta-bands and reactive changes in the all studied bands. The largest number of gender differences (by amount of absolute power in alpha- and beta-bands, amount of relative power in theta-band, reaction on eyes closing in the all studied bands) is observed in group of sportsmen practicing cyclic types of sport. Typical feature of sportswomen's cerebrum bioelectric activity is less evident reaction on eyes closing regardless of sport skill and of character of executed motions. At the same time sportsmen's neurofeedback successfulness is defined by three factors (sex, sport skill, general kinematic characteristic of executed motions) as a single no one of it don't have significant influencing. The received data partly explains why before authors didn't be able to reveal the differences of sportsmen's neurofeedback successfulness dependently on sport specialization (Tristan, 2005), skill (Cherapkina, 2010) and sex (Kal'sina, 2002), took separately. Obviously the revealed regularity dues to fact that typical EEG changes even if they marked by very significant in professional term sign have large individual variability and could show their influencing only in aggregate.

\section{CONCLUSIONS}

The held research allowed concluding that revealed changes of sportsmen's EEG depend on kinematic characteristic of executed motions, sport skill and sex. These factors influence on neurofeedback successfulness only in aggregate.

\section{REFERENCES}

1. BAIOVA N. The successfulness and efficiency the local alpha-stimulating training using of situational sport types sportsmen: author's abstract of dis. Cand Biol Scien. Tuymen; 2003.

2. BALAKHNICHEV V. Selection and training of track and field athletics sportswomen with sexual dimorphism account taken. Theory and practice of physical culture. 2007; 4:11-15.

3. BUGAIOV S, VODYANOY A, NIKITINA E. Conceptual training models of biofeedback method. Novosibirsk: Biofeedback - 2: The theory and practice. 1993; 37-40.

4. CHERAPKINA L, BAEVA N, TALAMOVA I, TRISTAN V. Using of neurofeedback in training and educational process. Theory and practice of physical culture. 2006; 1:12-14.

5. CHERAPKINA L. Dynamic of spectral characteristics of sportsmen heart rate who had neurofeedback course. Omsk: IX All-Russian scientific conference of Biofeedback in medicine and sport. 2009; 107-114.

6. CHERAPKINA L. Neurofeedback efficiency of different skilled sportsmen and persons actively practiced physical culture. Siberian physical education: scientific-methodological journal. 2010; 1(26):64-67.

7. CRIDER A. When relaxation backfires: A cognitive interpretation of relaxation-induced anxiety. Biofeedback. 1998; 1(26):22-23,33.

8. FEDOTCHEV A. About EEG-biofeedback efficiency using for functional stress-illnesses correction of patient. Human physiology. 2010; 1(36):100-105.

9. IORDANSKAYA F. Morphofunctional abilities of female during long-term adaptation to contemporary sport loads. Theory and practice of physical culture. 1999; 6:43-50.

10. KAL'SINA V. The influence of the sexual dimorphism on successfulness and efficiency of the local alpha-stimulating training of the sportsmen: Dis. Cand. Med. Scien. Kurgan; 2002.

11. KONARIOVA I. The changes of human frequency EEG pattern during a neurofeedback session. Neurophysiology. 2005; 5/6(37): 443-451.

12. LOPARIOV A. The EEG-FB course influence on certain electroencephalographic parameters of 
weightlifting sport type and fight sportsmen. Omsk: IX All-Russian scientific conference of Biofeedback in medicine and sport. 2009; 77-80.

13. MOHOVIKOVA I, SVYATOGOR I. The criterions of the biofeedback successfulness estimation of the cerebrum potentials. Moscow: VII All-Russian scientific conference of Biofeedback in medicine and sport. 2005; 27-31.

14. POGADAEVA 0 . The predictors of efficiency of the alpha-stimulating training using in sport: author's abstract of Dis. Cand. Biol. Scien. Tomsk; 2001.

15. SCHWARTZ M. The contemporary problems of biofeedback. Novosibirsk: Biofeedback - 3: The theory and practice. 1998; 14-24.

16. SOBOLEVA T. About problems of female sport. Theory and practice of physical culture. 1999; 6:56-63.

17. SOROKO S, MUSURALIEV T. The opportunities of directed EEG-parameters changes of human with the adoptive biofeedback using. Human physiology. 1995; 5(21):5-17.

18. STRIZHKOVA O, CHERAPKINA L, STRIZHKOVA T. The effects of neurofeedback using in the different training mesocycles of the gymnast-women. Omsk: IX All-Russian scientific conference of Biofeedback in medicine and sport. 2009; 93-98.

19. STYOPOCHKINA S, CHERAPKINA L, TRISTAN V. Brain bioelectricity activity of the sportsmen underwent the neurofeedback course. Bulletin of Siberian medicine. 2010; 2(9):83-87.

20. SVYATOGOR I, MOHOVICOVA I, BEKSHAEV S, FROLOVA T. The estimation of efficiency and successfulness of biofeedback method using for management of cerebrum potential. Biology feedback. 2000; 1:8-11.

21. SVYATOGOR I, MOHOVIKOVA I. Character of reorganizations of statistical structure of EEG components interactions in the course of neurofeedback. Bulletin of Siberian medicine. 2010; 2(9):53-58.

22. THOMAS M, DASGUPTA B, REYES E. Performance anxiety during biofeedback training. USA: Proceedings of the 22nd annual meeting of the AAPB. 1991; 75-78.

23. TRISTAN V, POGADAEVA O, CHERAPKINA L, TRISTAN V. The opportunity of the alphastimulating training using for sportsmen training. Novosibirsk: Biofeedback -4 : The theory and practice. 2002; 242-245.

24. TRISTAN VV. The neurophysiological mechanisms of neurofeedback successfulness of the different sport specialization sportsmen. Moscow: VII All-Russian scientific conference of Biofeedback in medicine and sport. 2005; 83-85.

25. VISOCHIN U, DENISENCO U, GORDEEV U. The increasing of functional abilities of sportsmen organism on base of biofeedback. Human physiology. 2005; 31(3):93-99. 